\title{
The Effect of Small Size Uterine Fibroids on Pregnancy Outcomes in High-risk Pregnancies
}

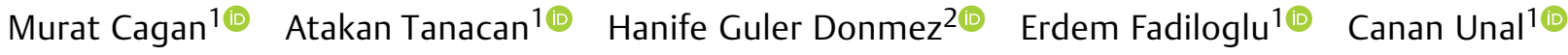 \\ Mehmet Sinan Beksac ${ }^{10}$ \\ ${ }^{1}$ Division of Perinatology, Department of Obstetrics and Gynecology, \\ Ankara, Turkey \\ ${ }^{2}$ Department of Biology, Hacettepe University, Ankara, Turkey \\ Address for correspondence Murat Cagan, Division of Perinatology, \\ Department of Obstetrics and Gynecology, Hacettepe University, \\ Sıhhıye, Ankara, Turkey \\ (e-mail: drmuratcagan@gmail.com).
} Rev Bras Ginecol Obstet 2020;42(9):535-539.

\begin{abstract}
Keywords

- uterine fibroid

- myoma

- pregnancy

- CS myomectomy
\end{abstract}

\section{Introduction}

Uterine fibroids (also known as myomas) are benign monoclonal smooth muscle neoplasms and are the most common pelvic tumors in women of reproductive age. The incidence of fibroids in pregnancy is from 3.3 to $10.7 \%^{1,2}$ Although most pregnancies in women with fibroids are uneventful, adverse pregnancy outcomes due to fibroids, such as miscarriage, preterm labor, placenta previa, placental abruption, fetal growth restriction, malpresentation, and peripartum hemorrhage, may occur in 10 to $30 \%$ of these patients. ${ }^{3}$ Moreover, the most common complications of fibroids during pregnancy are pain due to degeneration or torsion of the pedunculated fibroid, as well as pelvic pressure-related problems and vaginal bleeding. ${ }^{4,5}$ The frequency of major adverse outcomes correlates with the size of the fibroid and is especially high in women with fibroids $>5 \mathrm{~cm}$ in diameter. ${ }^{6,7}$

The majority of prospective studies using ultrasound to follow the size of uterine fibroids during pregnancy have shown that fibroid size remains stable ( $<10 \%$ change in size) from the pregestational period to the end of pregnancy. ${ }^{6}$ In spite of this, some studies report an increase in size during pregnancy. ${ }^{8}$ Besides, larger fibroids ( $>5 \mathrm{~cm}$ in diameter) are more likely to grow, whereas smaller fibroids are more likely to remain stable in size. ${ }^{4}$ Fibroids may cause pregnancy loss but there is a lack of consensus in the association between uterine fibroids and recurrent miscarriages among the medical community. ${ }^{9}$ Depending on the size and location, fibroids may alter the contour of the intrauterine cavity, leading to decidual atrophy or distortion of the vascular architecture of the decidua and affect implantation, placentation, and ongoing pregnancy. ${ }^{10}$ received

February 11, 2020

accepted

May 21, 2020
DOI https://doi.org/

10.1055/s-0040-1713913. ISSN 0100-7203.
Copyright $\odot 2020$ by Thieme Revinter

Publicações Ltda, Rio de Janeiro, Brazil
License terms

(c) (i) 
Myomectomy during the course of cesarean section (CS) is questionable because of an increased risk of intrapartum and short-term postpartum complications, especially bleeding. However, many authors agree that myomectomy is a safe procedure during CS. ${ }^{11}$ The recent literature advocates elective or opportunistic myomectomy in well-selected cases during CS. ${ }^{12}$

Although there were various studies about pregnancies with fibroids in the literature, the number of studies on the effect of small fibroids on pregnancy was limited. Therefore, in the present study, we aimed to evaluate the obstetric outcomes of singleton high-risk pregnancies with a uterine fibroid $<5 \mathrm{~cm}$.

\section{Methods}

The present retrospective cohort study was conducted among high-risk pregnant women who were followed-up by a single surgeon (M. S. B.) at the Division of Perinatology, Department of Obstetrics and Gynecology from the Hacettepe University Hospital between August 2016 and December 2019. Women with uterine anomaly, those with multiple pregnancies, and pregnant women with fibroids $>5 \mathrm{~cm}$ in diameter or multiple fibroids were excluded. In the remaining 172 patients, pregnant women with preconceptionally diagnosed small/medium size $(<5 \mathrm{~cm})$ single uterine fibroids (study group) were compared with pregnant women without uterine fibroids (control group). The required data were obtained from the patients' files and the electronic database of our institution. The study protocol was approved by the Ethics Committee of Hacettepe University with the reference number of GO 19/1064, and informed consent was obtained from all participants.

Pregnancies with poor obstetric history, chronic inflammatory diseases, autoimmune disorders, metabolic and/or inflammatory risk factors for placenta-mediated pregnancy complications were defined as high-risk pregnancy in this study. All high-risk pregnancies were included in a special antenatal care program for the optimal management of their risk factors. Pregnancy follow-up consisted of serial ultrasonography to evaluate fetal growth, aneuploidy screening (combined or triple test), fetal anatomy scanning at the $20^{\text {th }}$ to $24^{\text {th }}$ gestational weeks, oral glucose challenge test, and a non-stress test performed according to national and international guidelines. The iron supplement $(30 \mathrm{mg})$ was given to all pregnant women daily.

The study and control groups were compared in terms of maternal age, gravidity, parity, Beksac Obstetric Index pregnancy (BOIp), miscarriage rate, hemoglobin $(\mathrm{Hb})$ level at the first trimester, gestational age at birth, birth weight, $5^{\text {th }}$ minute Apgar score, fetal presentation, postdelivery $\mathrm{Hb}$ (8 hours after delivery), and delta $\mathrm{Hb}$ levels (the difference between the postdelivery and first trimester $\mathrm{Hb}$ levels). The BOI is a special obstetric index for the assessment of risk levels in pregnancies depending on their previous obstetric histories [(number of alive children $+(n / 10)) /$ Gravida]. The BOI value calculated in the preexisting pregnancy was defined as BOIp. ${ }^{13}$ Beksac Obstetric Index is used widely in the literature for the comparison of risk levels for different patient groups. This index is used in many studies regarding various types of maternal risk factors. ${ }^{14,15}$ Furthermore, characteristics of the uterine fibroid (size, type, and location), the growth rate of the fibroid during pregnancy, location of the placenta, obstetric complications due to fibroids, cesarean myomectomy rate, and delta fibroid size (the difference between the size of the fibroid during delivery and at the preconceptional period) were evaluated in the study group.

Statistical analyses were performed using the IBM SPSS statistics software, version 22.0 (IBM Corp., Armonk, NY, USA). Variables were investigated using visual (histograms, probability plots) and analytical methods (Shapiro-Wilk test) to determine the normality of distribution. As the data were not normally distributed, the Mann-Whitney U-test was performed for the comparison of continuous variables, and the chi-square test was performed for comparing categoric variables between the groups. A two-tailed $p$-value $<0.05$ was considered statistically significant.

\section{Results}

The present study included 172 high-risk pregnant women. There were 25 cases in the study group and 147 cases in the control group. Demographic features and clinical characteristics of both groups were summarized in - Table 1 .

Miscarriage rates were similar between the groups (16\% and $17 \%$ for the study and control groups, respectively $[p=0.84]$ ). There were no statistically significant differences between the groups in terms of gestational age at birth, birth weight, and $5^{\text {th }}$ minute Apgar score. There were no early preterm deliveries in the cohort. Five (20\%) and $32(21 \%)$ late preterm deliveries were detected in the study and control groups, respectively. Median BOIp was 0.657 for both groups $(p=0.858)$. Malpresentation rates were $4 \%$ and $4.1 \%$ for the study and control groups, respectively $(p=0.841)$. The number of patients who received a blood transfusion for postpartum anemia was $1(4 \%)$ in the study group and $2(1.4 \%)$ in the control group $(p=0.351)$.

Basic characteristics of the study group were presented in -Table 2. None of the uterine fibroids was located in the cervix. Topographic locations of uterine fibroids were subserosal (68\%), intramural (28\%) and submucosal (4\%). The mean size of uterine fibroids at preconception and birth was $2.16 \mathrm{~cm}( \pm 0.75)$ and $2.54 \mathrm{~cm}( \pm 0.77)$, respectively. Fifteen uterine fibroids ( $60 \%$ of the study group) were increased, and the others $(40 \%)$ were stable in size during pregnancy. The mean delta fibroid size was $0.37 \mathrm{~cm}( \pm 0.61)$.

Placental locations were anterior (32\%), posterior (48\%), and fundal (\%20) in the study group. Placenta previa and placental abruption were not shown in the study group. Five patients from the study group $(20 \%)$ had retroplacental myoma. Out of these five patients, only one was complicated with preterm delivery, and the remainders had no adverse pregnancy outcome. Miscarriage was shown in four cases in the study group. Two patients were hospitalized with pelvic pain due to degeneration of the fibroid, and three patients were hospitalized with vaginal bleeding in the first trimester. The frequency of preterm delivery was $20 \%$ among women 
Table 1 Comparison of demographic features and clinical characteristics between groups

\begin{tabular}{|c|c|c|c|}
\hline & $\begin{array}{l}\text { Study group }(n=25) \\
\text { (median, min-max) }\end{array}$ & $\begin{array}{l}\text { Control group }(n=147) \\
\text { (median, min-max) }\end{array}$ & $p$-value \\
\hline Maternal age & $35.00(18-41)$ & $33.00(20-42)$ & 0.055 \\
\hline Gravidity & $3.00(1-8)$ & $3.00(1-9)$ & 0.522 \\
\hline Parity & $2.00(1-5)$ & $2.00(1-5)$ & 0.819 \\
\hline BOIp & $0.657(0.22-1.31)$ & $0.657(0.15-1.31)$ & 0.858 \\
\hline Miscarriage rate $(n, \%)$ & $4(16 \%)$ & $25(17 \%)$ & 0.840 \\
\hline Hb level at $1^{\text {st }}$ trimester & $12.3(7.6-14.8)$ & $12.4(8.4-14.8)$ & 0.531 \\
\hline Gestational age at birth & $37.00(34-38)$ & $37.00(34-40)$ & 0.782 \\
\hline Birth weight & $2,930(2,300-3,720)$ & $2,970(810-4,310)$ & 0.602 \\
\hline $5^{\text {th }}$ minute Apgar score & $9.00(4-10)$ & $10.00(0-10)$ & 0.693 \\
\hline Postdelivery Hb level & $10.1(5.6-12.2)$ & $10.4(6.3-13.6)$ & 0.142 \\
\hline Delta-Hb level & $2.0(0.3-5.0)$ & $1.8(0.4-4.7)$ & 0.263 \\
\hline
\end{tabular}

Abbreviations: BOlp, Beksac Obstetric Index pregnancy; Hb, hemoglobin, min, minimum; max, maximum; n, number.

Table 2 Basic characteristics of the study group (25 pregnant women with uterine fibroids)

\begin{tabular}{ll}
\hline Parameter & N (\%) or mean \pm SD \\
\hline Uterine fibroid location & $15(60)$ \\
$\quad$ Anterior & $7(28)$ \\
Posterior & $3(12)$ \\
$\quad$ Fundal & \\
Uterine fibroid & \\
topographic location & $17(68)$ \\
$\quad$ Subserosal & $7(28)$ \\
Intramural & $1(4)$ \\
$\quad$ Submucosal & $2.16 \pm 0.75$ \\
Uterin fibroid size & \\
preconception (cm) & $2.54 \pm 0.77$ \\
Uterin fibroid size & \\
at birth (cm) & \\
Modes of delivery & 4 \\
Miscarriage & $2\left(9.5^{\ddagger}\right)$ \\
Vaginal delivery & $4\left(19^{\ddagger}\right)$ \\
CS without myomectomy & $15\left(71.4^{\ddagger}\right)$ \\
CS myomectomy &
\end{tabular}

Abbreviations: $\mathrm{CS}$, cesarean section; $\mathrm{N}$, number; SD, standard deviation. ${ }^{\ddagger}$ Rates are given after exclusion of cases with miscarriage.

with fibroids. In the study group, the CS rate was $90.5 \%$, and none of the CS was performed due to uterine fibroids. Myomectomy was performed during CS in 15 cases (71.4\%).

\section{Discussion}

Uterine fibroids are the most common benign uterine tumors, with an estimated incidence of 20 to $40 \%$ in women during their reproductive years. The association of myoma and pregnancy is becoming more frequent due to the ad- vanced maternal age. ${ }^{16}$ In our study, $14.5 \%$ of 172 pregnant women had uterine fibroids with a diameter $<5 \mathrm{~cm}$.

Previous studies have shown a possible association between fibroids and increased risk of adverse pregnancy outcomes. ${ }^{17-19}$ In 2008 , Klatsky et al ${ }^{18}$ reported an increased risk of miscarriage in women with uterine fibroids compared with women without fibroids. According to a study conducted by Navid et $\mathrm{al}^{20}$ the frequency of miscarriage among women with fibroids was $10 \%$. In our study, we found that miscarriage rates were $16 \%$ and $17 \%$ in women with and without myomas, respectively, most probably due to the characteristics of our cases. Fetal malpresentation has also been reported to be more common among women with fibroids. Klatsky et $\mathrm{ll}^{18}$ reported a frequency of malpresentation of $16 \%$ among women with fibroids, $\sim 2.5$ times higher than in the general population. Similar results have been reported by Navid et $\mathrm{al}^{20}$ In our study, malpresentation rates were $4 \%$ and $4.1 \%$ for the study and control groups, respectively. This may be explained by the inclusion of the patients with a smaller size of fibroids. This shows us that small fibroids do not affect the malpresentation rate. Shavell et $\mathrm{al}^{21}$ showed that compared with women with no fibroids or small fibroids $(\leq 5 \mathrm{~cm})$, women with large fibroids ( $>5 \mathrm{~cm}$ ) delivered at a significantly earlier gestational age (38.6 versus 38.4 versus 36.5 weeks). According to our results, the preterm delivery rate was $20 \%$ in the study group, and there was no statistically significant difference between the groups in terms of gestational age at birth (median gestational age was 37.0 weeks for both groups). Placental abruption has been associated with uterine fibroids and seems to be related to fibroid location. ${ }^{22}$ Placental abruption and placenta previa were not observed in our study group, most probably due to the size of the fibroids. Fetal growth does not appear to be affected by the presence of uterine fibroids, which we have also demonstrated in our study. ${ }^{22}$ Degeneration occurs in around $10 \%$ of pregnant women with fibroids. ${ }^{4}$ Likewise, two patients (8\%) were hospitalized with pelvic pain due to degeneration in our study and none of these pregnancies complicated with any adverse event. 
Risk factors for pregnancy complications appear to be the size and the location of fibroids, such as the large size of over $5 \mathrm{~cm}$ and retroplacental location and/or distortion of the uterine cavity. ${ }^{17-23}$ In our study, we have demonstrated that fibroids $<5 \mathrm{~cm}$ did not provide an additional risk in terms of adverse pregnancy outcomes. The risk of uterine fibroidrelated complications during pregnancy might be primarily correlated with the size of myomas.

Pregnancy-related increases in steroid hormone levels and uterine blood flow affect fibroid growth. ${ }^{4}$ Aharoni et $\mathrm{al}^{24}$ reported leiomyomas to be mostly unchanged during pregnancy (59\%). It has also been reported that the size of the fibroids was increased in $22 \%$ of the patients and the growth percentage of these fibroids was found to be $25 \%{ }^{24}$ In our study, the size of the fibroids was increased in $60 \%$ of the cases, and the growth percentage of the fibroids was also $25 \%$.

Song et $\mathrm{a}^{25}$ reviewed 9 case-control studies that included more than 1,000 women with fibroids, of whom $41 \%$ underwent cesarean myomectomy and 59\% underwent CS alone. They could not demonstrate any difference between groups in terms of safety parameters. ${ }^{25}$ Turgal et al ${ }^{11}$ found no statistical difference in the adhesion formations between women who had previously undergone cesarean myomectomy for small fibroids and controls who had not undergone myomectomy during their previous CS. Cesarean sections of our patients were performed due to other obstetrical indications, and we have demonstrated that opportunistic cesarean myomectomy was convenient as a safe and viable option in well-selected cases. Thus, we may conclude that cesarean myomectomy may be safely performed in patients with a myoma $<5 \mathrm{~cm}$ by experienced physicians. However, appropriate case selection must still be performed and previously reported complications must be kept in mind.

The limitations of this study were the relatively small number of cases, retrospective design, and lack of information related to fibroid sizes throughout gestational trimesters. On the other hand, longitudinal follow-up of the cases and presentation of single surgeon experience are the strengths of this study.

\section{Conclusion}

In conclusion, small size uterine fibroids $(<5 \mathrm{~cm})$ seem to have no adverse effect on pregnancy outcomes even in highrisk pregnancies, and cesarean myomectomy may be safely performed in properly selected cases.

\section{Contributors}

All of the authors contributed with the project and the interpretation of the data, with the writing of the article, the critical review of the intellectual content, and with the final approval of the version to be published.

\section{Conflict of Interests}

The authors have no conflict of interests to declare.

\section{References}

1 Laughlin SK, Baird DD, Savitz DA, Herring AH, Hartmann KE. Prevalence of uterine leiomyomas in the first trimester of pregnancy: an ultrasound-screening study. Obstet Gynecol. 2009;113 (03):630-635. Doi: 10.1097/AOG.0b013e318197bbaf

2 Stout MJ, Odibo AO, Graseck AS, Macones GA, Crane JP, Cahill AG. Leiomyomas at routine second-trimester ultrasound examination and adverse obstetric outcomes. Obstet Gynecol. 2010;116 (05):1056-1063. Doi: 10.1097/AOG.0b013e3181f7496d

3 Vitale SG, Tropea A, Rossetti D, Carnelli M, Cianci A. Management of uterine leiomyomas in pregnancy: review of literature. Updates Surg. 2013;65(03):179-182. Doi: 10.1007/s13304013-0198-z

4 Ezzedine D, Norwitz ER. Are women with uterine fibroids at increased risk for adverse pregnancy outcome? Clin Obstet Gynecol. 2016;59(01):119-127. Doi: 10.1097/grf.0000000000000169

5 Yalınkaya A, Kangal K, Güzel AI, Erdem S. Successful myomectomy of a giant myoma during pregnancy. Gynecol Obstet Reprod Med. 2010;16:194-195

6 Vitagliano A, Noventa M, Di Spiezio Sardo A, Saccone G, Gizzo S, Borgato $S$, et al. Uterine fibroid size modifications during pregnancy and puerperium: evidence from the first systematic review of literature. Arch Gynecol Obstet. 2018;297(04):823-835. Doi: 10.1007/s00404-017-4621-4

7 Rice JP, Kay HH, Mahony BS. The clinical significance of uterine leiomyomas in pregnancy. Am J Obstet Gynecol. 1989;160(5 Pt 1):1212-1216. Doi: 10.1016/0002-9378(89)90194-4

8 Rosati P, Exacoustòs C, Mancuso S. Longitudinal evaluation of uterine myoma growth during pregnancy. A sonographic study. J Ultrasound Med. 1992;11(10):511-515. Doi: 10.7863/ jum.1992.11.10.511

9 Russo M, Suen M, Bedaiwy M, Chen I. Prevalence of uterine fibroids among women with two or more recurrent pregnancy losses: a systematic review. J Minim Invasive Gynecol. 2016;23 (05):702-706. Doi: 10.1016/j.jmig.2016.03.018

10 Diamond MP, Polan ML. Intrauterine synechiae and leiomyomas in the evaluation and treatment of repetitive spontaneous abortions. Semin Reprod Med. 1989;7(02):111-114. Doi: 10.1055/s2007-1021389

11 Turgal M, Ozgu-Erdinc AS, Beksac K, Ozyuncu O, Karaagaoglu E, Beksac MS. Myomectomy during cesarean section and adhesion formation as a long-term postoperative complication. Ginekol Pol. 2015;86(06):457-460. Doi: 10.17772/gp/2404

12 Chauhan AR. Cesarean myomectomy: necessity or opportunity? J Obstet Gynaecol India. 2018;68(06):432-436. Doi: 10.1007/ s13224-018-1114-8

13 Beksaç MS, Aydın E, Tuğral M, Karaağaoğlu E. An obstetrics index for the assessment of risk levels of "high risk pregnancy" groups. Gynecol Obstet Reprod Med. 2016;21(01):10-13

14 Beksac K, Tanacan A, Cagan M, et al. Relationship of cholelithiasis and urolithiasis with methylenetetrahydrofolate reductase polymorphisms. J Investigative Surg. 2020

15 Tanacan A, Beksac MS, Orgul G, Duru S, Sener B, Karaagaoglu E. Impact of extractable nuclear antigen, anti-double stranded DNA, antiphospholipid antibody, and anticardiolipin antibody positivity on obstetrical complications and pregnancy outcomes. Hum Antibodies. 2019;27(02):135-141. Doi: 10.3233/HAB-180359

16 Krimou Y, Erraghay S, Guennoun A, Mamouni N, Bouchikhi C, Banani A. Myoma praevia and pregnancy. Pan Afr Med J. 2019; 33:216. Doi: 10.11604/pamj.2019.33.216.14898

17 Ouyang DW, Economy KE, Norwitz ER. Obstetric complications of fibroids. Obstet Gynecol Clin North Am. 2006;33(01):153-169. Doi: 10.1016/j.ogc.2005.12.010

18 Klatsky PC, Tran ND, Caughey AB, Fujimoto VY. Fibroids and reproductive outcomes: a systematic literature review from conception to delivery. Am J Obstet Gynecol. 2008;198(04): 357-366. Doi: 10.1016/j.ajog.2007.12.039 
19 Parazzini F, Tozzi L, Bianchi S. Pregnancy outcome and uterine fibroids. Best Pract Res Clin Obstet Gynaecol. 2016;34:74-84. Doi: 10.1016/j.bpobgyn.2015.11.017

20 Navid S, Arshad S, Qurat-ul-Ain, Meo RA. Impact of leiomyoma in pregnancy. J Ayub Med Coll Abbottabad. 2012;24(01): 90-92

21 Shavell VI, Thakur M, Sawant A, Kruger ML, Jones TB, Singh M, et al. Adverse obstetric outcomes associated with sonographically identified large uterine fibroids. Fertil Steril. 2012;97(01):107-110. Doi: 10.1016/j.fertnstert.2011.10.009

22 Coronado GD, Marshall LM, Schwartz SM. Complications in pregnancy, labor, and delivery with uterine leiomyomas: a popu- lation-based study. Obstet Gynecol. 2000;95(05):764-769. Doi: 10.1016/s0029-7844(99)00605-5

23 Vitale SG, Padula F, Gulino FA. Management of uterine fibroids in pregnancy: recent trends. Curr Opin Obstet Gynecol. 2015;27 (06):432-437. Doi: 10.1097/gco.0000000000000220

24 Aharoni A, Reiter A, Golan D, Paltiely Y, Sharf M. Patterns of growth of uterine leiomyomas during pregnancy. A prospective longitudinal study. Br J Obstet Gynaecol. 1988;95(05):510-513. Doi: 10.1111/j.1471-0528.1988.tb12807.x

25 Song D, Zhang W, Chames MC, Guo J. Myomectomy during cesarean delivery. Int J Gynaecol Obstet. 2013;121(03):208-213. Doi: 10.1016/ j.ijgo.2013.01.021 